\title{
In Situ Analysis of Solvent/Nonsolvent Exchange and Phase Separation Processes During the Membrane Formation of Polylactides
}

\author{
P. VAN DE WITTE, ${ }^{1, *}$ I. W. A. VAN DEN BERG,' I. FEIJEN, ${ }^{1}$ J. L. REEVE, ${ }^{2}$ and A. J. MCHUGH ${ }^{2}$ \\ 'Department of Chemical Technology and Institute for Biomedical Technology, University of Twente, PO Box 217, \\ $7500 \mathrm{AE}$ Enschede, The Netherlands, and ${ }^{2}$ Department of Chemical Engineering, University of Illinois \\ Urbana-Champaign, Urbana, Illinois 61801
}

\begin{abstract}
SYNOPSIS
Membrane formation of polylactides has been studied using in situ analysis techniques. An experimental method based on the use of dark ground optics and reflected light illumination is used to monitor the mass transfer and phase separation dynamics during formation. Additionally, the phase separation and structure formation has been studied using optical microscopy. The results of the dark ground optics technique for the polymer/solvent/ nonsolvent systems poly-L-lactide/chloroform/methanol and poly-DL-lactide/chloroform/ methanol showed that the diffusion kinetics were similar for the semicrystalline poly-Llactide (PLLA) and the amorphous poly-DL-lactide. The influence of the molecular weight of the polymers on the diffusion kinetics was found to be negligible. Increasing the polymer concentration of the casting solution decreased the rate of diffusion. The phase separation of poly-DL-lactide was studied with optical microscopy and found to proceed via liquidliquid demixing. For poly-L-lactide solutions of relatively low concentration $(5-6 \% \mathrm{w} / \mathrm{w})$, phase separation proceeded via liquid-liquid demixing followed by crystallization. For more concentrated PLLA solutions, phase separation proceeded directly via solid-liquid demixing processes. Additionally, for $6 \% \mathrm{w} / \mathrm{w}$ solutions of poly-L-lactide in dioxane immersed in methanol, precipitation also occurred solely via solid-liquid demixing. (C) 1996 John Wiley \& Sons, Inc.
\end{abstract}

\section{INTRODUCTION}

Phase transitions play a very important role in the preparation of membranes by the immersion precipitation process. The final morphology of a membrane is the result of these phase transitions. ${ }^{1}$ A great deal of research has been dedicated to determining the role of the various phase transitions during immersion precipitation. However, due to the complexity of the process, studies have been restricted mainly to analysis of resulting membrane morphologies and determination of the phase boundaries of the corresponding ternary systems.

\footnotetext{
* To whom correspondence should be addressed. Journal of Applied Polymer Science, Vol. 61, 685-695 (1996) (C) 1996 John Wiley \& Sons, Inc. CCC 0021-8995/96/040685-11
}

The complicated mass transfer processes occurring after immersion of the polymer solution in the nonsolvent greatly inhibit analysis of the structure formation. Recently, progress has been made in mathematical models which predict composition paths in the solution as a function of time and space coordinates after immersion of the polymer solution in the nonsolvent. ${ }^{2,3}$ Unfortunately, the models are only valid up until the initial point of phase separation. Additionally, complications arising from the input parameters of the model prevent a quantitative description of mass transfer.

Surprisingly, very few experimental studies on the in situ mass transfer and phase separation have been published. ${ }^{4-16}$ In the present work the results of an experimental study on the solvent/nonsolvent exchange and the structure formation of polylactide 


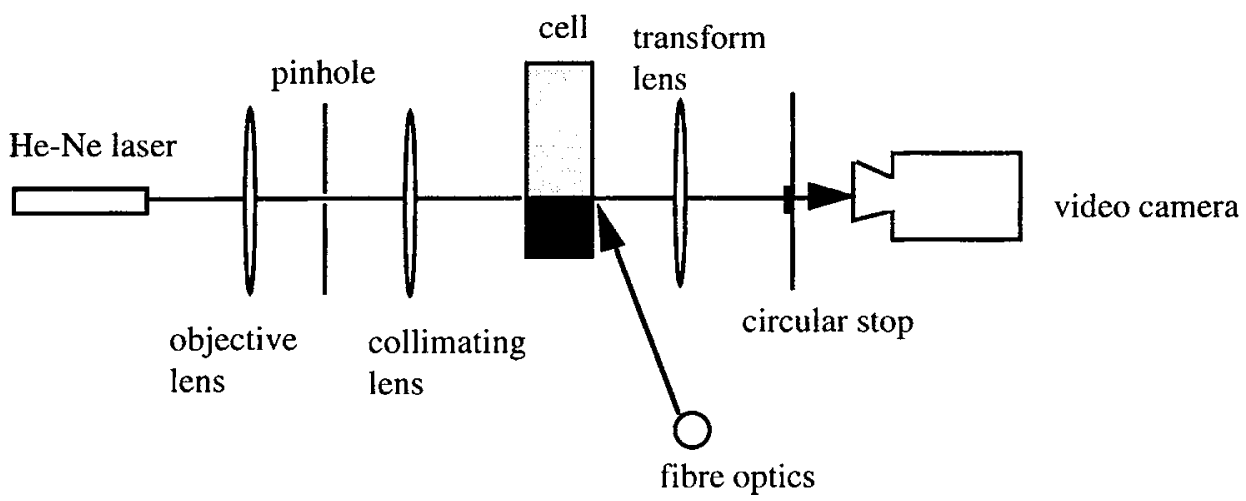

Figure 1 schematical representation of the optical setup for study of mass transfer processes in the film.

solutions will be presented. For mass transfer experiments, the optical setup recently described by McHugh and Tsay was used. ${ }^{8,13}$ The technique used most frequently for studying phase separation processes during immersion precipitation involves placing a polymer solution between two glass slides and putting these in an optical microscope. . $^{4,5,10,11}$ The nonsolvent is then placed in contact with the periphery of the cover slide and subsequently drawn to the polymer solution between the slides by capillary forces. Usually no details of the phase separation processes can be observed due to the large sample thickness. However, in the present study an improved optical technique will be presented that allows visualization of greater detail.

\section{EXPERIMENTAL}

\section{Materials}

Polymer solutions were prepared using poly-L-lactide (PLLA) and poly-DL-lactide (PDLLA) samples obtained from Purac Biochem B.V., Gorinchem, The Netherlands. For optical microscopy, samples were used with an intrinsic viscosity of $2 \mathrm{dL} / \mathrm{g}$ (chloroform, $25^{\circ} \mathrm{C}$ ). For the solvent/nonsolvent exchange, PLLA and PDLLA fractions were used with intrinsic viscosities of 0.8 and $4.6 \mathrm{dL} / \mathrm{g}$. Solvents were of analytical grade (Merck, Darmstadt, Germany).

\section{Optical Setup for Solvent-Nonsolvent Exchange}

The details of the dark ground optical setup for monitoring mass transfer and phase separation in the polymer solution during the quench have been described in more detail elsewhere. ${ }^{8,13}$ The diffusion cell which contains the polymer solution is made of optical-quality fused silica (Helma Cells, Inc.). The cavity containing the solution has an optical path length of $4 \mathrm{~mm}$ and is of variable depth $(4 \mathrm{~mm}$ for diffusion experiments and $3 \mathrm{~mm}$ for gelation). An upper chamber, made of aluminum, contains the nonsolvent. The two chambers are initially separated by a thin metal foil. Interfacial contact is achieved by removal of the separating plate using a motor-driven device.

A schematic of the optical setup is shown in Figure 1. Light from the laser source is spatially filtered by passing it through an objective lens, pinhole aperture, and collimating lens to produce an expanded noiseless beam of uniform intensity. The gradient in refractive index caused by concentration gradients that form during the solvent/nonsolvent diffusive exchange induces a phase shift in the light passing through the cell. The retardance at any position and time is given by

$$
\delta(Y, t)=\frac{2 \pi w\left[n(Y, t)-n_{R}\right]}{\lambda}
$$

where $Y$ is the position in the polymer solution relative to the initial interface, $n(Y, t)$ is the solution refractive index, $w$ is the cell thickness, $\lambda$ is the wavelength of light $(632.8 \mathrm{~nm})$, and the reference index, $n_{R}$, is that of the initially uniform composition in the polymer solution. The diffraction image of the phase-modulated beam emerging from the cell is filtered on the back focal plane of the transform lens with a circular stop to remove the unrefracted light image. The resultant dark ground image is focused through the camera for recording of the fringe pattern which is superimposed on the cell. The re- 
lationship between the phase shift $(\delta)$ and the fringe pattern intensity $(I)$ will be given by

$$
I(Y, t)=4 C^{2} \sin ^{2}\left[\frac{\delta(Y, t)}{2}\right]
$$

where $C$ is a constant associated with the optical elements.

The dark ground image allows observation of this intensity distribution near the propagating diffusion front and, depending on conditions, distinguishes fingering instabilities. A second optical light source, directed at the cell from the camera side, reflects light due to turbid areas in the solution which may be two-phase fluid (liquid-liquid) regions or solidified gel. Video images showing the combined diffraction pattern and two-phase regions are recorded by the video camera. For analysis, the images are frozen at a given time and digitized by the computer, and then the positions of the fringes are determined by finding the minimum intensity of a fringe on screen using video-imaging software. Additionally, this video-imaging software is used to monitor the motion of the phase separation front.

\section{Optical Setup for Visualization of Phase Separation Processes}

Details of the phase separation processes after immersion were studied using an optical microscope, as shown in Figure 2. Solutions were injected in small flat capillaries of $0.4 \mathrm{~mm}$ thickness (Camslides, Camlab, Cambridge GB). Very viscous solutions were pushed in the capillary using a Pasteur pipet glued to the capillary and applying air pressure. Using a syringe with a thin needle, nonsolvent was injected on top of the solution. The processes occurring at the interface and the gel front were then studied using a Leitz-Ortolux microscope equipped with polarizers.

\section{RESULTS AND DISCUSSION}

\section{Solvent-Nonsolvent Exchange during Immersion Precipitation}

Only the solvent-nonsolvent system chloroformmethanol was evaluated in this study. For most experiments, low-molecular-weight polylactide samples $([\eta]=0.8 \mathrm{dL} / \mathrm{g})$ were used to enable analysis over a wide concentration range. In Figure 3 a series of digitized images of the diffusion fringe

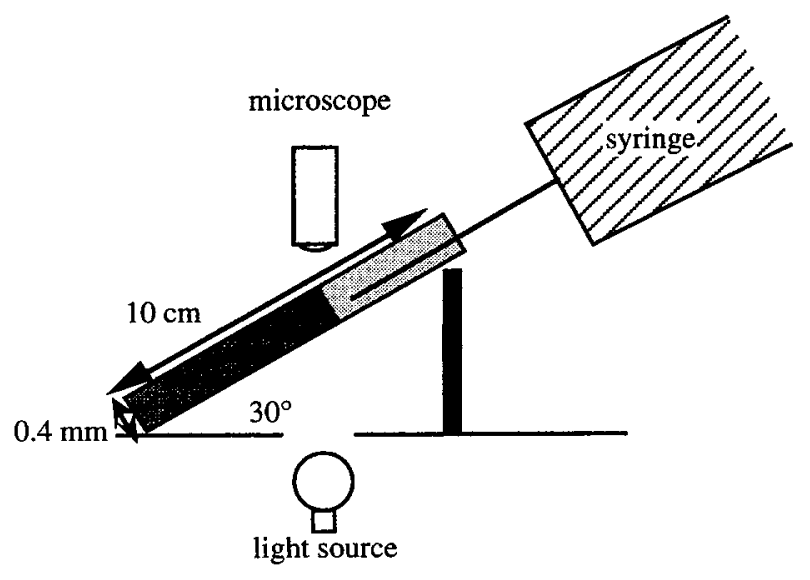

Figure 2 Optical setup for studying phase separation processes.

patterns as a function of time are presented for a $5 \% \mathrm{w} / \mathrm{w}$ solution of PDLLA in chloroform. Time zero is taken when half of the cell is in contact with the coagulation bath. The fringes move gradually from the interface to the bottom of the cell. Location of the first fringe minimum, $Y$, with respect to the bath-film interface is also illustrated. All systems studied gave nice diffraction patterns with up to 10 discernable fringes that were uniformly parallel.

The bath-film interface was seen to move down with time. This was noticeable as residual polymer which was seen to coagulate on the cell walls. The polymer solutions eventually phase separated. The phase separation was not uniform or consistent and did not lend well to measurement, due in part to the film shrinkage and the poor quality of the gel that formed. Additional experiments will be detailed in a later section that achieved more well-behaved gelation behavior by forcing the system closer to the miscibility gap.

The position of the first fringe is a measure of the penetration depth of the nonsolvent in the polymer solution. Because the refractive indexes of the polylactides $(n \approx 1.45)$ are comparable with the refractive index of chloroform $(n \approx 1.48)$, the refractive index of the polymer solution does not change much with composition and the propagation of the fringe patterns for the two polylactides and for the various polymer concentrations are directly comparable ( $n_{\text {methanol }} \approx 1.33$ ).

In Figure 4 the square of the position of the first fringe in the solution relative to the initial position of the interface is presented as a function of time for different PLLA concentrations. The plots are 

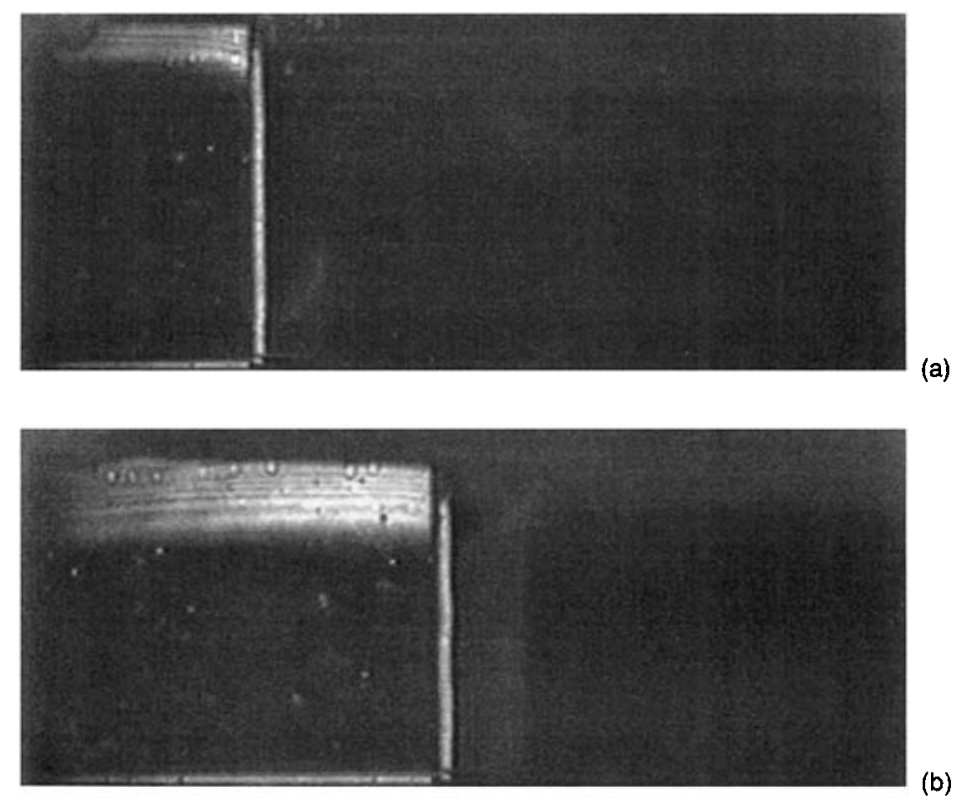

(b)

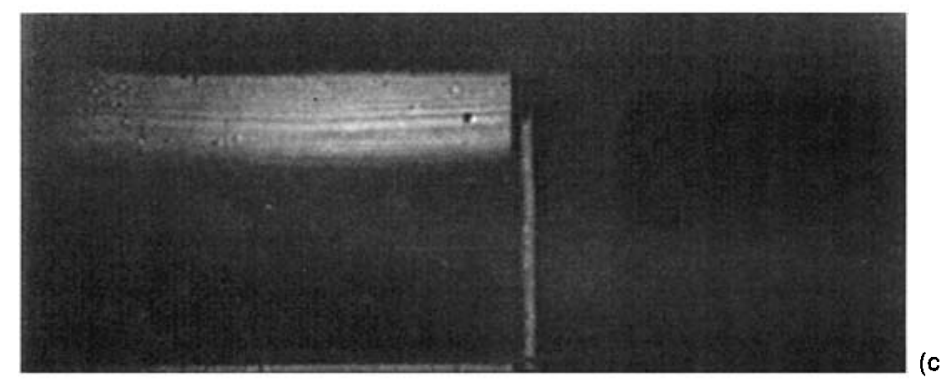

(c)

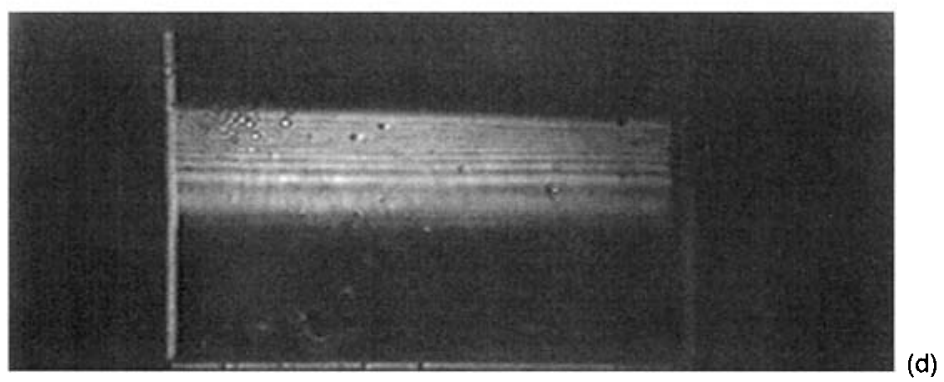

Figure 3 Digitized images of the fringe patterns obtained for a $5 \% \mathrm{w} / \mathrm{w}$ solution of PDLLA in chloroform; the nonsolvent is methanol. The coordinate $(Y)$ is also indicated. (a) Time: $-30 \mathrm{~s}$ (the cell is not yet positioned in the middle of the screen), (b) time: $5 \mathrm{~s}$; (c) time: $1 \mathrm{~min}$; (d) time: $2 \mathrm{~min}$; (e) time: $4 \mathrm{~min}$; (f) time: $6 \mathrm{~min}$.

initially linear, as has also been found by Tsay and McHugh for cellulose acetate systems and by Miller in his studies of polyethersulfone (PES) solutions. ${ }^{8,13-16}$ This linear behavior is also predicted by the diffusive mass transfer equations. ${ }^{2,3}$ The slope of this linear region, which corresponds to the rate of mass transfer, decreases with increasing polymer concentration. In Miller's study it was re- ported that for PES the slopes of these lines $\left(S_{1}\right)$ vary linearly with the initial polymer volume fraction of the polymer solution $(\phi): S_{1}=M_{1} \phi+B_{1}$ ( $M_{1}$ and $B_{1}$ are constants). In the present study similar behavior was found to exist in the polylactides. Diffusion experiments were all performed at least twice to ensure reproducibility, and Figure 5 shows the average value of the slope of diffusion 

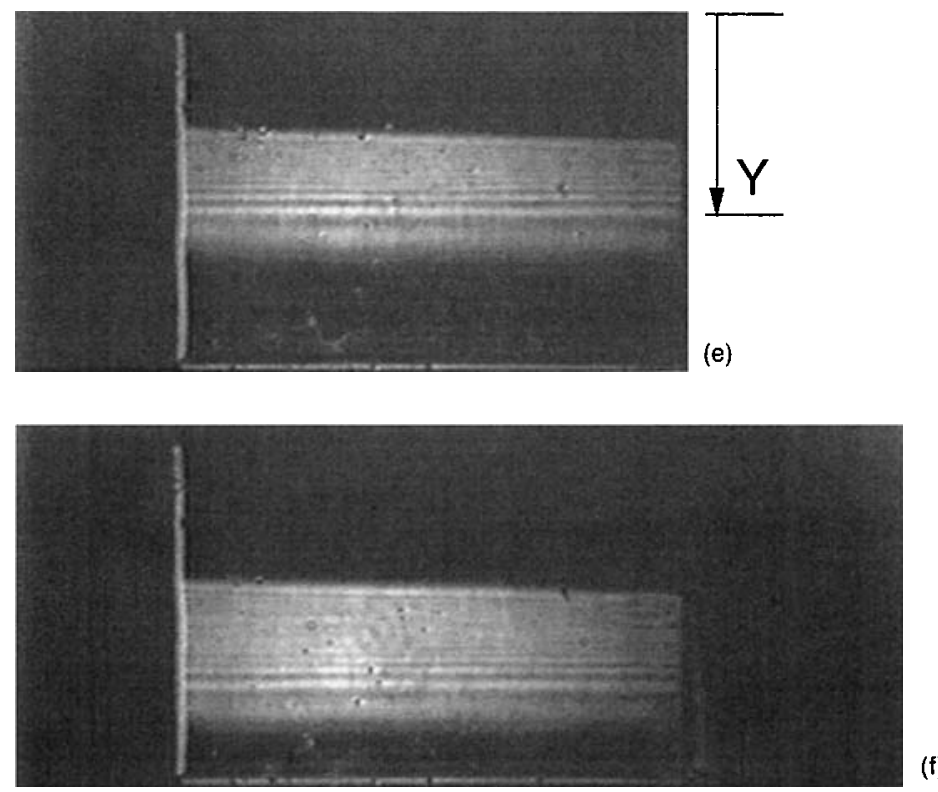

Figure 3 (Continued from the previous page)

front motion versus initial polymer concentration for both PLLA and PDLLA.

Figure 5 shows that in the range we investigated, the rate of mass transfer for a given initial polymer concentration is independent of the stereochemical

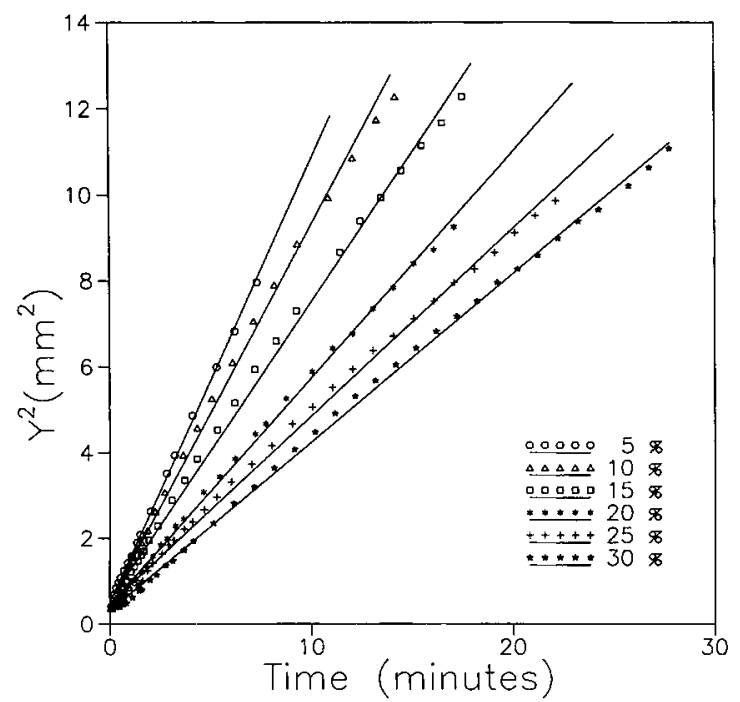

Figure 4 Squared position of the first fringe in the cell relative to the position of the initial interface as a function of time and concentration for the system poly-L-lactidechloroform-methanol. Notice that the intercept at the $y$ axis can vary somewhat for the different systems due to differences in appointment of time zero. The intrinsic viscosity of PLLA $=0.8 \mathrm{dL} / \mathrm{g}$. configuration of the polylactide (i.e., PLLA vs. PDLLA). This confirms that during the immersion precipitation process, the solvent/nonsolvent exchanges for PLLA and PDLLA are comparable. This is in line with the fact that under similar conditions the differences in precipitation times are generally small for low-concentration polylactide/chloroform solutions quenched in methanol. ${ }^{17}$ However, even though the mass transfer is comparable, liquid-liq-

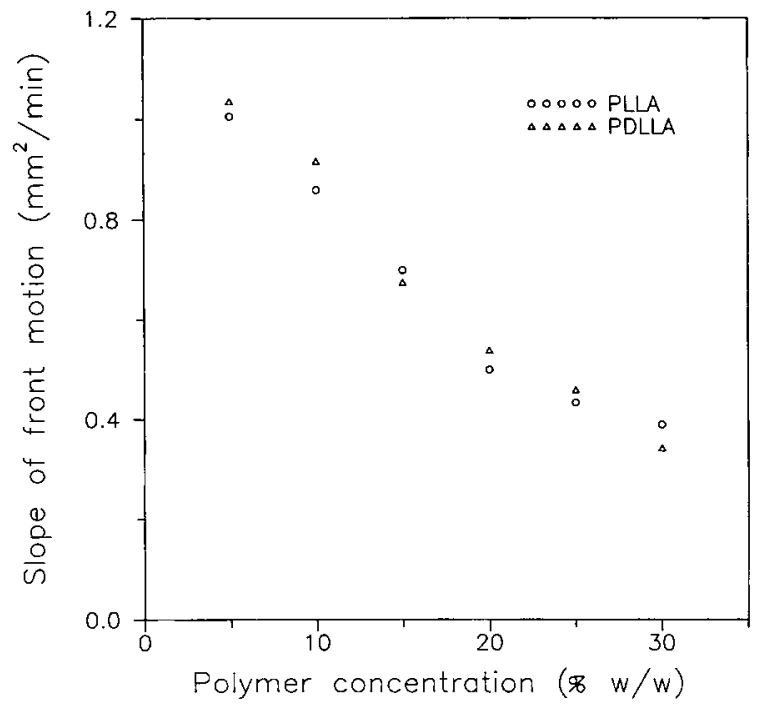

Figure 5 Average values of the slope of diffusion front motion versus initial polymer concentration for PLLA and PDLLA. 


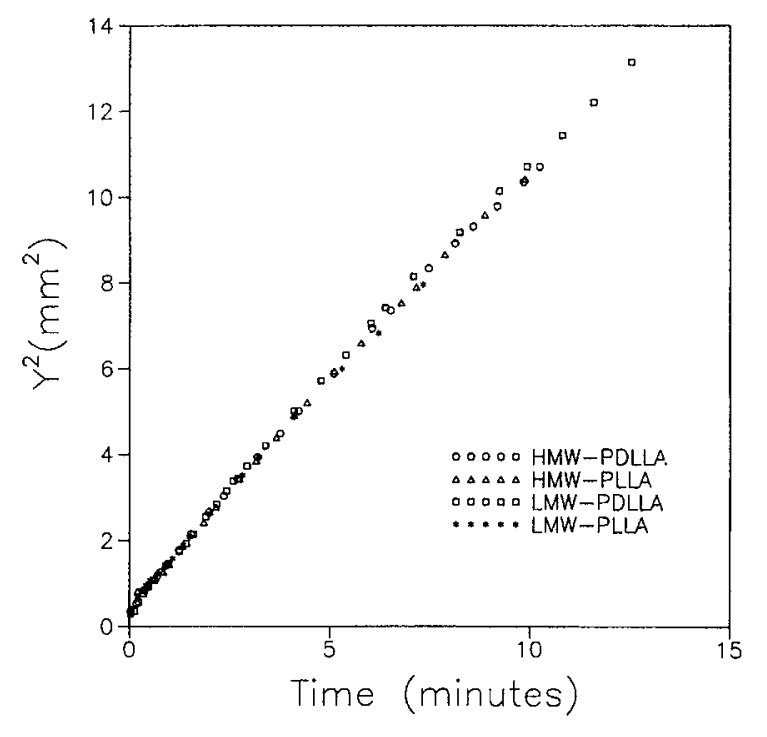

Figure 6 Influence of molecular weight of PDLLA and PLLA on the diffusion kinetics (for further details, see legend for Fig. 4). HMW: $4.6 \mathrm{dL} / \mathrm{g}$; LMW: $0.8 \mathrm{dL} / \mathrm{g}$.

uid demixing did not play a large role during the immersion precipitation of concentrated solutions $(>20 \% \mathrm{w} / \mathrm{w})$ of PLLA in chloroform but does for PDLLA. ${ }^{17-20}$ This result emphasizes that the membrane morphology that results is largely determined by the type of phase transitions that a system can undergo.

Another series of experiments was performed to investigate the influence of molecular weight on the mass transfer dynamics by using polymer samples with intrinsic viscosities of 0.8 and $4.6 \mathrm{dL} / \mathrm{g}$. Five and ten percent $w / w$ samples of both PLLA and PDLLA in chloroform were quenched with methanol, and the resulting diffusion fringe motion was measured. The results for $5 \% \mathrm{w} / \mathrm{w}$ are shown in Figure 6. This figure indicates that the differences in rates of propagation of diffusion fronts are negligible within the experimental error of our system for the two molecular weights at the concentrations investigated. This phenomenon has been observed in other instances - for example, in the system PLLAdioxane-water it was observed that for intrinsic viscosities between 1.6 and $4.7 \mathrm{dL} / \mathrm{g}$, the delay time for demixing for cast films of a $7 \% \mathrm{w} / \mathrm{w}$ solution remained the same despite a 100 -fold difference in the viscosity of the solution (experiments not shown). The macroscopic viscosity increases rapidly with increasing molecular weight because the polymer chains get more entangled. The distance between the entanglements does not change much with mo- lecular weight. This is why, in general, the diffusion coefficient of small molecules in low-concentration polymer solutions does not depend on the molecular weight of the polymer and in fact is almost the same as the binary diffusion coefficient. These experiments confirm that from the viewpoint of solvent/ nonsolvent exchange, it is acceptable to compare membrane morphologies obtained using polymers with different molecular weights.

Additional experiments were performed to investigate the phase separation behavior of polylactides. Due to the low nonsolvent power of methanol, it took a long time for gelation to occur. To circumvent this and study gelation phenomena, 15\% w/w PLLA solutions were doped with varying amounts of methanol to push the initial composition closer to the binodal. This becomes clear from the gel growth data, as displayed in Figure 7. Experiments were all performed three times, and the slope of the linear region was found to be reproducible within 5\%. At increasing methanol concentrations in the casting solution, the gel starts growing earlier, and the gel growth is more uniform. Whether liquid-liquid demixing or solid-liquid demixing processes are responsible for the formation of the gels cannot be determined from this experiment. This gelation behavior is similar to what Miller found for PES, for which the slope of the linear region was found to be a linear function of the concentration, just as it is for diffusion front motion. ${ }^{14,15}$ Similarly, for poly-

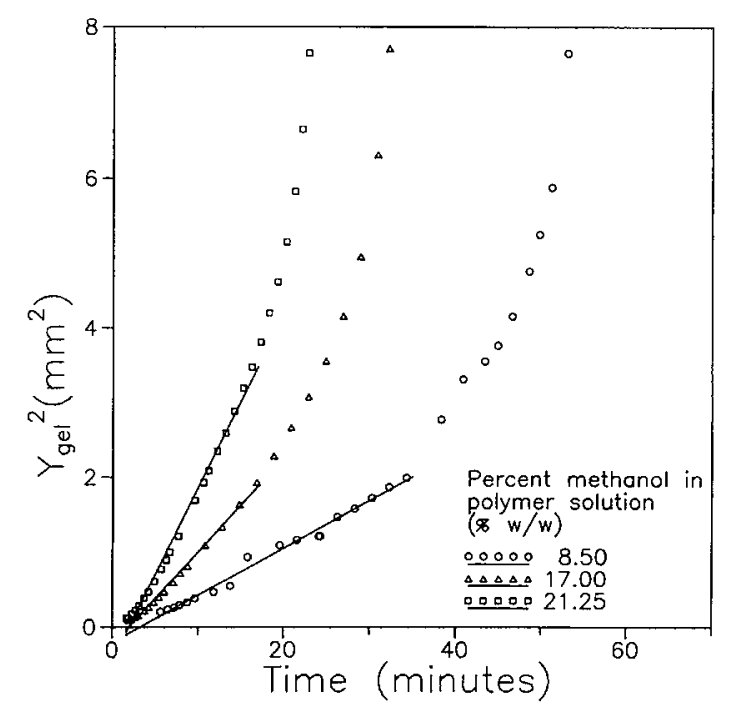

Figure 7 The position of the first fringe relative to the position of the initial interface for $15 \% \mathrm{w} / \mathrm{w}$ solutions of PLLA in chloroform/methanol mixtures (nonsolvent: methanol). 

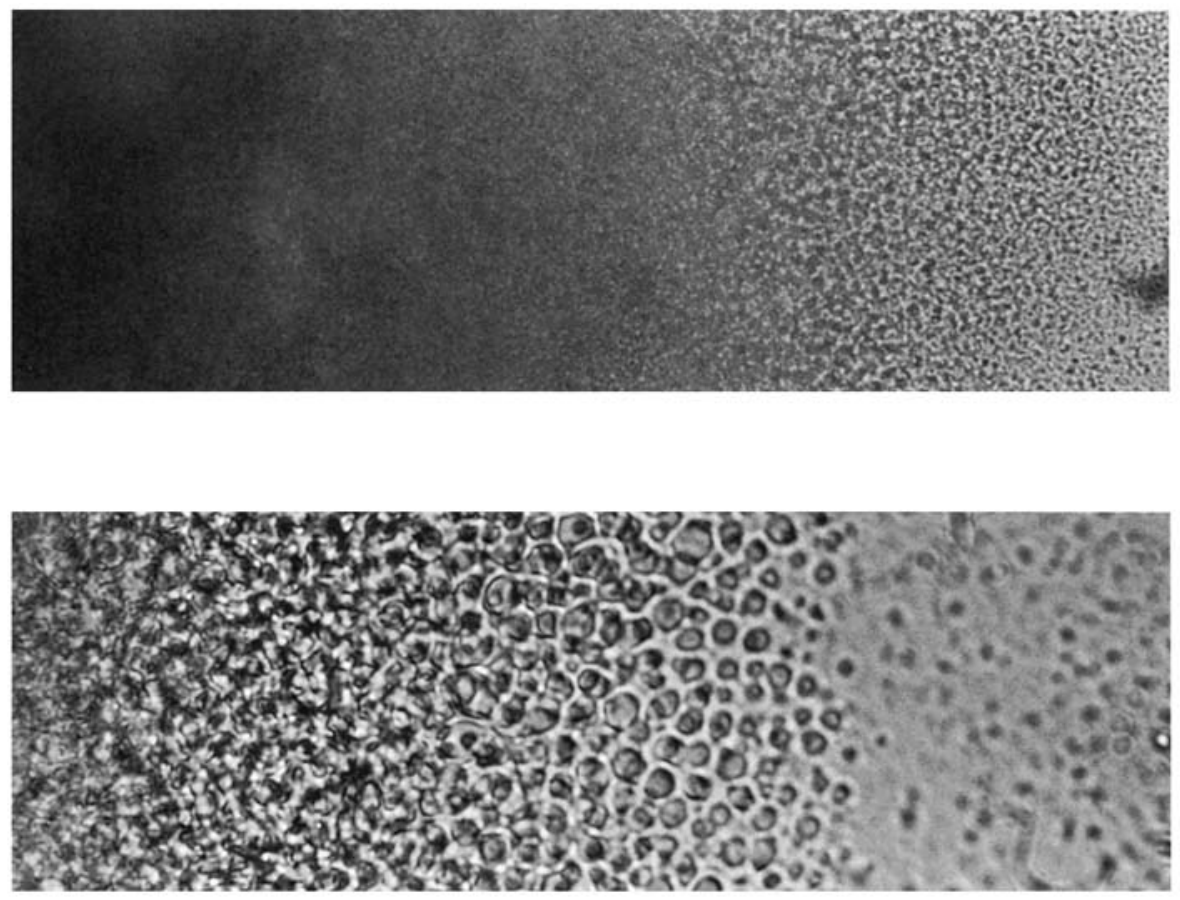

(b)

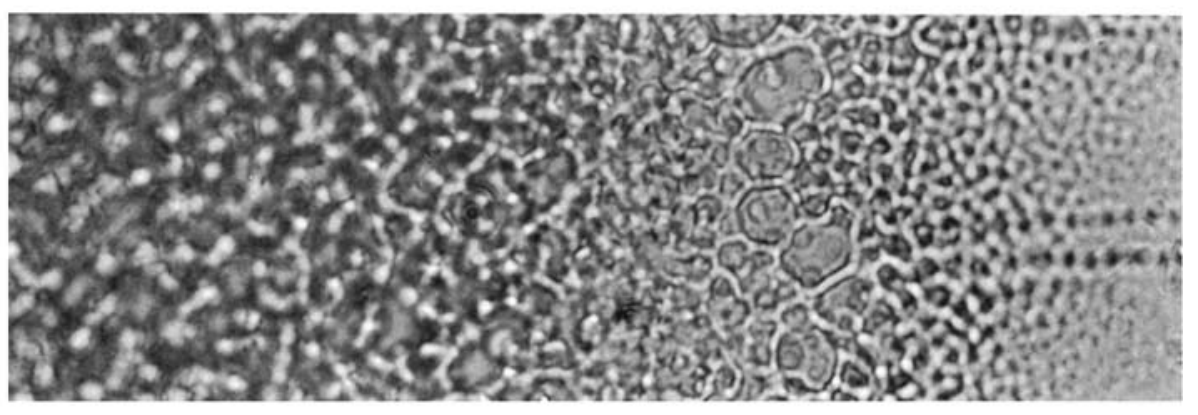

(c)

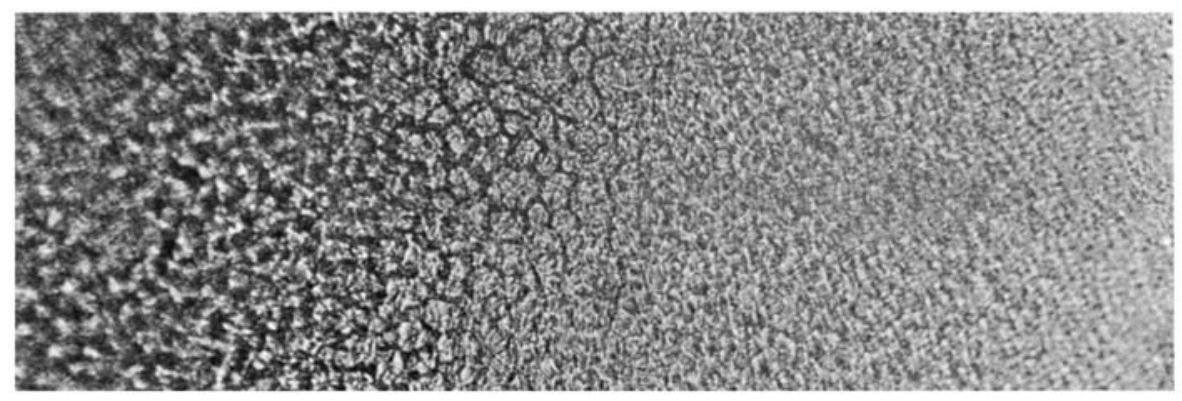

(d)

Figure 8 Micrographs of the phase separation processes occurring in the polymer solution after addition of the nonsolvent. The polymer solution is located on the right-hand side. The solution bath interface is located on the left-hand side but is not visible on the micrograph. (a) $20 \% \mathrm{w} / \mathrm{w}$ PDLLA in chloroform; nonsolvent: methanol (magnification $100 \times$ ). (b) $10 \% \mathrm{w} / \mathrm{w}$ PDLLA in chloroform; nonsolvent: methanol (magnification 250×). (c) $5 \%$ w/w PDLLA in chloroform; nonsolvent: methanol (magnification 250X). (d) $6 \% \mathrm{w} / \mathrm{w}$ PDLLA in dioxane; nonsolvent: water (magnification $250 \times$ ). 


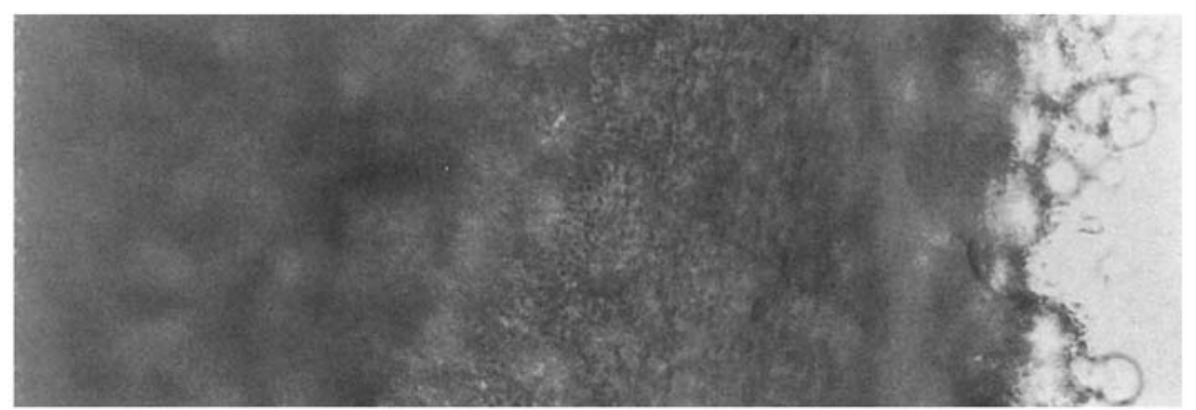

(a)

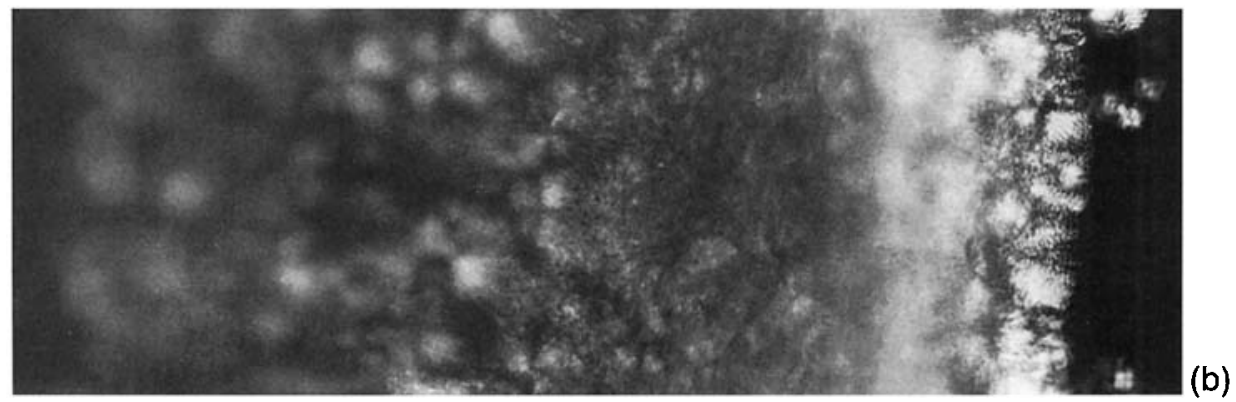

Figure 9 Micrographs of the phase separation processes occurring in the polymer solution after addition of the nonsolvent. The polymer solution is located on the right-hand side. The solution bath interface is located on the left-hand side but is not visible on the micrograph. (a) $20 \% \mathrm{w} / \mathrm{w}$ PLLA in chloroform; nonsolvent: methanol (unpolarized light, magnification $100 \times$ ). (b) $20 \% \mathrm{w} / \mathrm{w}$ PLLA in chloroform; nonsolvent: methanol (polarized light, magnification $100 \times$ ).

lactides there is a dependance of this slope on the percentage of dopant in the initial polymer solution which is approximately linear with the nonsolvent concentration.

\section{Visualization of Structure Formation during Immersion Precipitation}

Figure 8 presents micrographs of the phase separation processes occurring in PDLLA solutions shortly after addition of the nonsolvent. As expected, for solutions containing the amorphous poly-DLlactide, phase separation always occurred by liquidliquid demixing independent of solvent/nonsolvent combination and polymer concentration. A turbid gel layer was present close to the interface. The sample thickness is still too large to allow visualization of details of the processes in the gel layer. Just behind the gel layer in the solution, the nucleation and growth of droplets can clearly be observed. As expected, no crystallites could be detected using polarized light. To the best of our knowledge, micrographs with these details have not been published yet in the literature of the immersion precipitation process.
For the semicrystalline poly-L-lactide system, liquid-liquid demixing and solid-liquid demixing processes could be distinguished easily by visualization of the phase-separated solution with both polarized light and unpolarized light. For concentrated solutions of PLLA ( $>15 \% \mathrm{v} / \mathrm{v})$, the thickness of the gel layer observed with polarized light was the same as the thickness of the gel layer observed with unpolarized light. This indicates that liquid-liquid demixing does not precede solid-liquid demixing in these cases. An example is presented in Figure 9.

The spherulites are easily distinguished at the interface between the precipitated solution and the homogeneous solution. In Figure 10, micrographs are presented for a $5 \% \mathrm{v} / \mathrm{v}$ solution of PLLA in chloroform with methanol as a nonsolvent. The white area in the micrograph obtained with crossed polarizers indicates the presence of a crystalline gel. In the micrograph taken with unpolarized light, a second layer due to liquid-liquid phase separation can be observed between the gel layer and the homogeneous solution. This indicates that crystallization occurs in the polymer-rich phase generated by the liquid-liquid demixing process. 

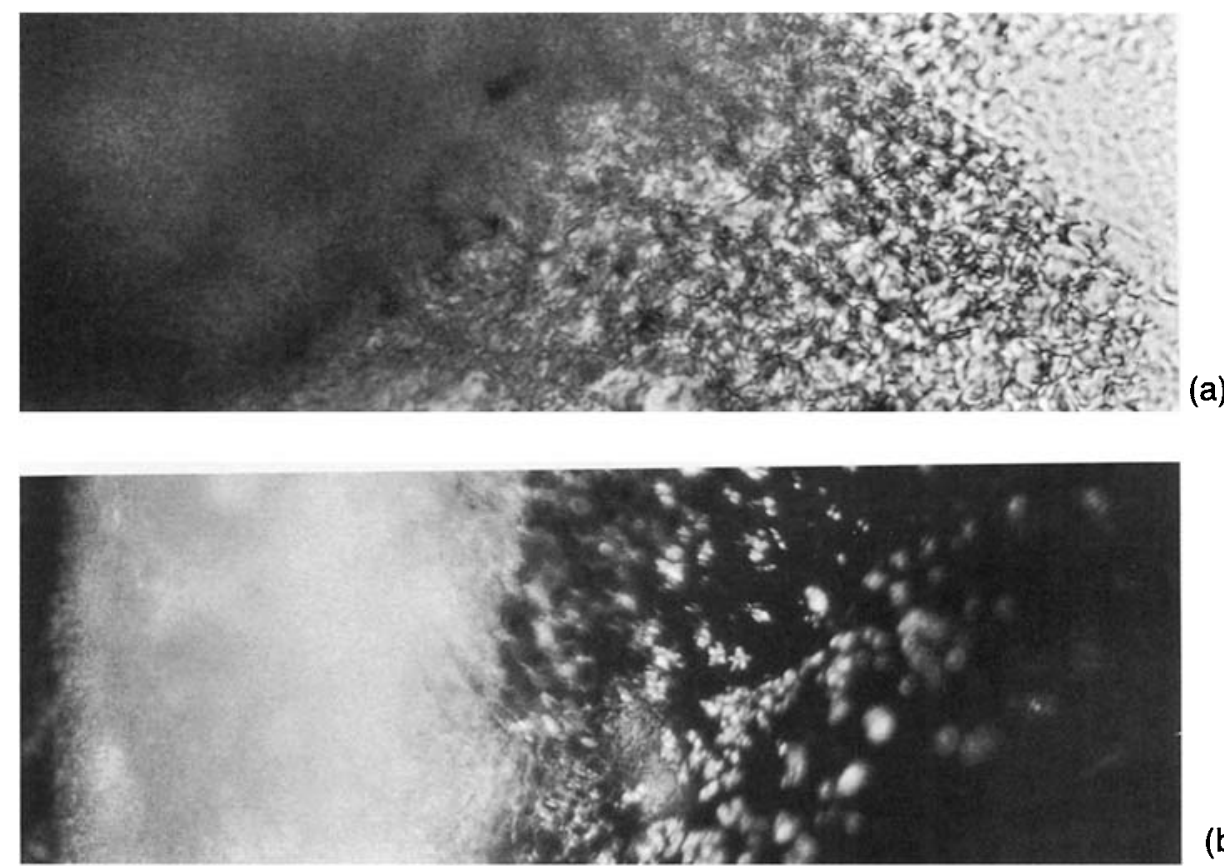

(b)

Figure 10 Micrographs of the phase separation processes occurring in the polymer solution after addition of the nonsolvent. The polymer solution is located on the right-hand side. The solution bath interface is located on the left-hand side but is not visible on the micrograph. (a) 5\% PLLA in chloroform; nonsolvent: methanol (unpolarized light, magnification 250X). (b) 5\% PLLA in chloroform; nonsolvent: methanol (polarized light, magnification $250 \times$ ).

Similar trends were observed with the solventnonsolvent system dioxane-water [Fig. 11(a,b)]. The corresponding system with dioxane-methanol did not show a layer due to liquid-liquid demixing. A homogeneous birefringent gel layer was detected [Fig. 11(c)].

These findings are in line with previous results for the phase diagrams and the resulting morphologies of the membranes obtained with these systems. ${ }^{17-20}$ For PDLLA, only evidence for liquid-liquid demixing was observed for all solvent/nonsolvent systems. For PLLA in combination with dioxane/water or chloroform/methanol at low polymer concentrations, liquid-liquid demixing preceded solid-liquid demixing and at high polymer concentration crystallization occurred without the preceding liquid-liquid demixing. For PLLA/dioxane/ methanol, liquid-liquid demixing processes did not play a role during the precipitation. It can be expected that this technique can be of use for other fundamental studies on membrane formation (e.g., macrovoid formation). Further improvements can be attained by further reducing the thickness of the capillary and by introducing video analysis tech- niques like in the system used for the study of solvent/nonsolvent exchange.

\section{CONCLUSIONS}

The results for the exchange studies show that diffusion in the systems with PLLA and PDLLA is comparable and independent of the molecular weight. The exchange rate decreases with increasing polymer concentrations in the casting solution. The phase separation behavior of simple PLLA-chloroform cast solutions proved to be difficult to measure, because of large film shrinkage and weak gel structure. However, cast solutions doped with nonsolvent displayed uniform, reproducible behavior and showed an increase in the rate of phase separation as the amount of dopant increased. The microscopic technique yielded useful additional information on the structure formation. For PDLLA-containing solutions, precipitation occurred by liquid-liquid demixing. For PLLA-containing solutions, depending on the solvent/nonsolvent combination and the com- 


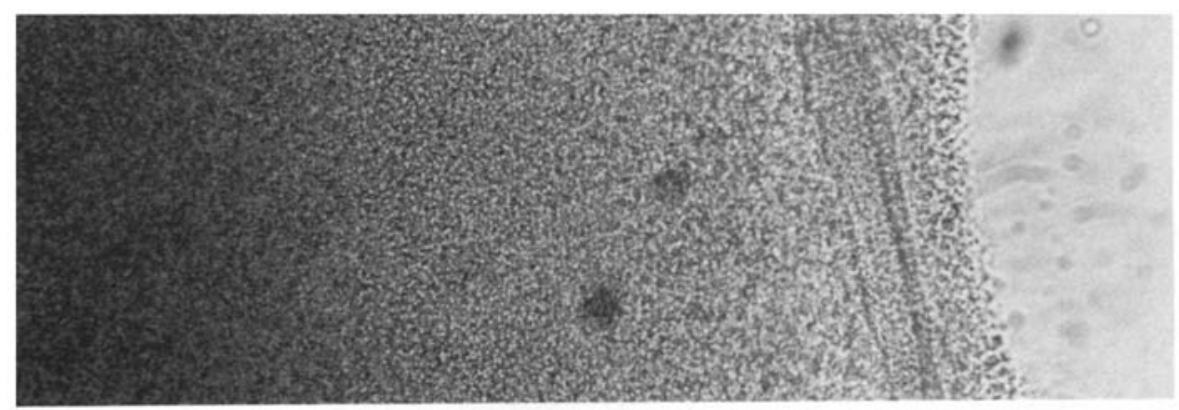

(a)

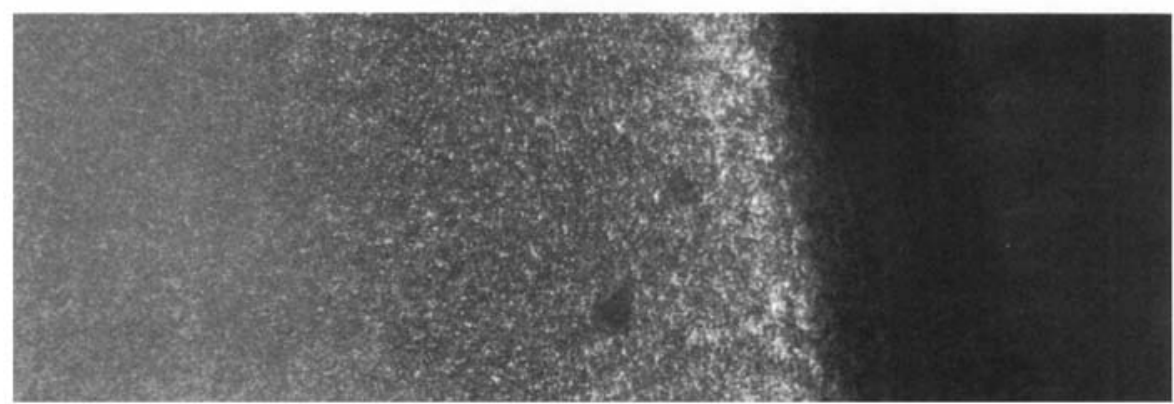

(b)

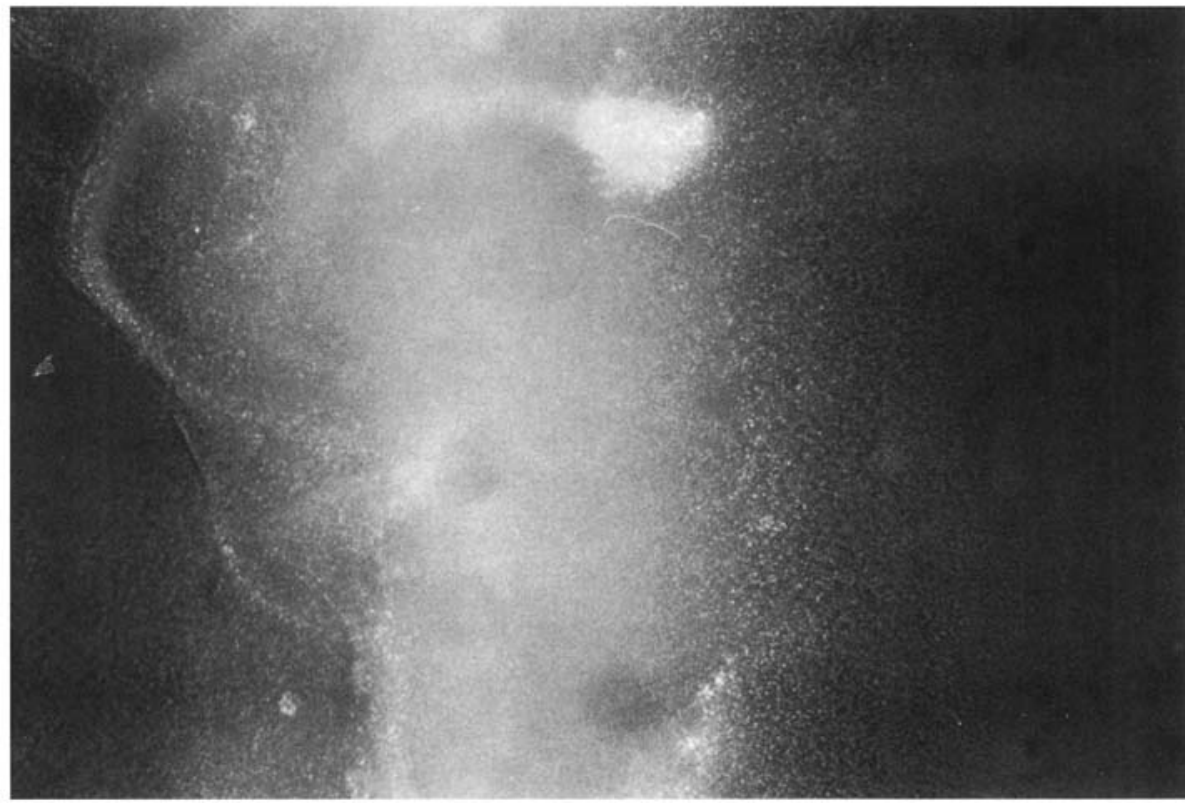

(c)

Figure 11 Micrographs of the phase separation processes occurring in the polymer solution after addition of the nonsolvent. The polymer solution is located on the right-hand side. The solution bath interface is located on the left-hand side but is visible only in the bottommost micrograph (c). (a) 6\% w/w PLLA in dioxane; nonsolvent: water (unpolarized light, magnification $100 \times$ ). (b) $6 \% \mathrm{w} / \mathrm{w}$ PLLA in dioxane; nonsolvent: water (polarized light, magnification $100 \times$ ). (c) $6 \% \mathrm{w} / \mathrm{w}$ PLLA in dioxane; nonsolvent: methanol (polarized light, magnification $100 \times)$.

position of the solution, both liquid-liquid demixing and solid-liquid demixing could occur. For low concentrated solutions of PLLA in dioxane and chloroform immersed in water and methanol, re- spectively, crystallization occurred after liquidliquid demixing. For concentrated solutions for the system PLLA/chloroform/methanol and low concentrated solutions for the system PLLA/ 
dioxane /methanol, precipitation occurred mainly by crystallization.

\section{REFERENCES}

1. M. Mulder, Basic Principles of Membrane Technology, Kluwer Academic Publishers, Dordrecht, The Netherlands, 1992.

2. A. J. Reuvers, J. W. A. van den Berg, and C. A. Smolders, J. Membrane Sci., 34, 45 (1987).

3. C. S. Tsay and A. J. McHugh, J. Polymer Sci. Part B: Pol. Phys. Ed., 28, 1327 (1990).

4. (a) M. A. Frommer and D. Lancet, in Reverse Osmosis Membrane Research, A. K. Londsdale and H. E. Podall, Eds., Plenum Press, New York, 1972; (b) M. Frommer and R. M. Messalem, Ind. Eng. Chem. Prod. Res. Develop, 12, 328 (1973).

5. L. Broens, D. M. Koenhen, and C. A. Smolders, Desalination, 22, 205 (1977).

6. F. Altena, J. Smid, J. W. A. van den Berg, J. G. Wijmans, and C. A. Smolders, Polymer, 26, 1531 (1985).

7. A. Viallat and S. Perez, J. Polymer Sci. Part B: Pol. Phys. Ed., 31, 1567 (1993).

8. A. J. McHugh and C. S. Tsay, J. Appl. Polym. Sci., 46, 2011 (1992).

9. G. E. Gaides and A. J. McHugh, J. Membrane Sci., 74, 830 (1992).
10. D. M. Koenhen, M. H. V. Mulder, and C. A. Smolders, J. Polymer Sci., 21, 1991 (1977).

11. H. Strathmann, K. Kock, P. Amar, and R. W. Baker, Desalination, 16, 179 (1975).

12. L. Ambrosone, G. Guarino, R. Sartorio, and V. Vitagliano, J. Membrane Sci., 45, 299 (1989).

13. C. S. Tsay and A. J. McHugh, Ind. Eng. Chem. Res., 31, 449 (1992).

14. D. C. Miller, M.S. thesis, University of Illinois, Urbana, 1993.

15. A. J. McHugh and D. C. Miller, J. Membrane Sci., 105, 121 (1995).

16. A. J. McHugh, Presentation at North American Membrane Society Annual Meeting, Breckenridge, Colorado, 1994.

17. P. van de Witte, H. Esselbrugge, P. J. Dijkstra, J. W. A. van den Berg, and J. Feijen, J. Membrane Sci., 113, 223 (1996).

18. P. van de Witte, P. J. Dijkstra, J. W. A. van den Berg, and J. Feijen, Polymer Preprints, 35, 842 (1994).

19. P. van de Witte, H. Esselbrugge, A. M. P. Peters, P. J. Dijkstra, J. Feijen, R. J. J. Groenewegen, J. Smid, J. Olijslager, J. M. Schakenraad, M. J. D. Eenink, and A. P. Sam, J. Controlled Release, 24, 61 (1993).

20. P. van de Witte, Ph.D. thesis, University of Twente, Enschede, The Netherlands, 1994.

Received June 26, 1995

Accepted October 1, 1995 